2B and/or US EPA Group C and higher). Logistic regression was used to calculate odds ratios (ORs) and 95\% confidence intervals (CIs). Models were adjusted for age, province, and use of a proxy respondent.

Results Nearly 20 "probably" and 50 "possibly" carcinogenic pesticides were reportedly used by participants. Men who used any "probably" carcinogenic pesticide had increased odds for NHL (OR $=1.63,95 \%$ CI: $1.23-2.16)$ and MM (OR $=1.56$, 95\% CI: $1.12-2.18$ ), but not for STS (OR $=1.13$; 95\% CI: $0.81-1.58)$ and HL (OR $=0.99,95 \%$ CI: $0.66-1.48)$ relative to men who did not use these pesticides. Similarly, men who used any "possibly" carcinogenic pesticide had higher odds for NHL (OR $=1.54,95 \%$ CI: $1.21-1.96)$ and $\mathrm{MM}(\mathrm{OR}=1.36$, 95\% CI: $1.02-1.81)$, but not for STS (OR $=1.02$, 95\% CI: $0.77-1.35)$ and HL (OR $=0.97,95 \%$ CI: $0.70-1.33)$.

Conclusions The use of any carcinogenic pesticide was associated with modest increases in odds for NHL and MM, but not for STS and HD. The ORs were slightly larger from use of "probably" compared to "possibly" carcinogenic pesticides. These results are consistent with IARC and US EPA pesticide classifications.

\section{RISK OF MAJOR LYMPHOMA SUBTYPES AND USE OF MOBILE PHONES}

P C Cocco, Satta, D'Andrea, Ursi, Nonne, Pilleri, Avataneo, Pau, Campagna. University of Cagliari, Monserrato, Italy

10.1136/oemed-2013-101717.163

Introduction Recent case-control studies have suggested an increase in risk of non Hodgkin Lymphoma (NHL) among mobile phone users. We explored the association in a case-control study conducted in Sardinia Italy in 1999-2004. Methods. Three hundred twenty two adult (age range 25-75) cases, first diagnosed with lymphoma along the study period, and 422 controls, randomly selected from population Registrars, frequency matched to cases by age, gender and local health unit of residence, participated to the study. In person interviews gathered information on data and age of purchase of a mobile telephone and duration of its daily use. We conducted unconditional logistic regression analysis in 322 lymphoma cases and 446 population controls, adjusting by age, gender and education.

Results Risk of lymphoma (all types; OR $=1.5$; 95\%CI 1.0 $2.1)$, and particularly chronic lymphocytic leukaemia $(\mathrm{OR}=1.8$; $95 \%$ CI 1.0 - 3.4) was elevated in subjects reporting use of mobile phones, but it decreased with duration of use, and it was more elevated for the most recent purchases and for age at first purchase $^{3} 56$ years.

Conclusions Our findings contradict some assumptions about the association between use of mobile phones and cancer risk. Information bias possibly played a role Overall, our study cannot provide support to the aetiological nature of the observed associations.

\section{CANCER INCIDENCE IN A COHORT OF BIOLOGY RESEARCH LABORATORY WORKERS IN THE NETHERLANDS}

M Hauptmann, Heemsbergen, Mooij, van Leeuwen. Netherlands Cancer Institute, Amsterdam, Nederland

10.1136/oemed-2013-101717.164
Objectives Research laboratory personnel is exposed to a wide variety of carcinogenic agents. The link between biological research work and a possible increased cancer risk has been studied in several European countries. We examined the incidence of cancer among persons employed in Dutch biology research laboratories, particularly cancer of the pancreas, brain, breast, and lung and non-Hodgkin's lymphoma.

Methods In a historical cohort study, 7307 laboratory workers employed in four Dutch institutions between 1960 and 1992 were followed for incidence of cancer and mortality from 1989 to 2009 based on linkage with the Netherlands Cancer Registry. Informationon the agents used in the research laboratories was obtained by a questionnaire sent to 2536 participants (64\% response) and by another questionnaire completed by 98 laboratory heads. Cancer incidence was compared with the general population via standardised incidence ratios (SIR). Internal comparisons of laboratory workers with a control group of 2404 unexposed employees of the same institutions were based on Cox regression.

Results During follow-up (mean duration, 16.6 years), 809 cancers were observed among exposed and unexposed cohort members, which affords $80 \%$ power to detect a SIR of 1.1 for all cancers among the laboratory workers and a hazard ratio of roughly 1.3 for laboratory workers compared with the unexposed group. Analyses by duration of employment, type of research lab, and job title are ongoing. Results on cancer mortality until 1995 have been previously published (Cancer Causes Control 2004;15(1):55-66). No increased cancer mortality risks were observed compared to the general population. However, based on internal comparisons, risks were elevated for several sites, particularly lung cancer.

Conclusions Strength of this cohort are the long follow-up and large size, including a sizeable control group. This allows external comparisons of cancer incidence with the general Dutch population as well as internal comparisons with similar, however unexposed, workers.

\section{BREAST AND CERVICAL CANCER SCREENING UTILISATION AMONG INSURED FEMALE EMPLOYEES AT A LARGE US COMPANY}

S E Starks, Kreckmann, Le, Symons. DuPont, Newark, United States of America

\subsection{6/oemed-2013-101717.165}

Objectives Female employees enrolled in a company-sponsored health insurance plan are eligible to receive preventive care benefits. We examined the utilisation of recommended screening tests for breast and cervical cancer among female employees of a large U. S. company.

Methods Using health insurance claims data, we identified female employees who were continuously enrolled from 2009 through 2011. The prevalence of biennial screening mammography among employees aged 40 to 64 years and the prevalence of Pap tests in the past three years among employees aged 21 to 64 years were calculated for groups defined by demographic and work characteristics.

Results Among 3,972 female employees aged 40 to 64 years, $62 \%$ had at least one screening mammography in 2010 or 2011 . Screening mammography utilisation did not differ substantially by race, marital status, pay-type or work location. Screening mammography utilisation was higher among employees aged 50 to 64 years $(65 \%)$ compared to those aged 40 to 49 years $(57 \%)$ and was higher among employees with day work schedules (64\%) compared to those with rotating work schedules (51\%). 